\title{
Clinicopathological Profile of Rhabdomyosarcoma in Children
}

\author{
UH BEGUM ${ }^{\mathrm{a}}$, A ISLAM ${ }^{\mathrm{b}}$
}

Summary:

Objectives: To describe the clinical profile as well as histopathological sub-types of Rhabdomyosarcoma in children.

Methods: A hospital base prospective observational study was conducted among 20 diagnosed cases of Rhabdomyosarcoma in children, those attending in Hemato-Oncology department of Bangabandhu Sheikh Mujib Medical University (BSMMU), Dhaka in the period between January to December 2009.

Results: The peak incidence of Rhabdomyosarcoma was in 1-5 years of age group ( $n=9,45 \%)$ with mean age 6.83 years with male to female ratio 5.66:1. The common sites of primary tumor was in head and neck region $(40 \%, n=08)$, followed by genito-urinary tract, $30 \%(n=06)$, extremities $20 \%(n=04)$, trunk $10 \%(n=02)$. The most common clinical presentation was mass lesion $100 \%(n=20)$, followed by local pain 25\% (n=05), urinary obstructions $15 \%(n=03)$

\section{Introduction:}

Rhabdomyosarcoma (RMS) is the most common soft tissue sarcoma in children. ${ }^{1}$ Although Weber first described Rhabdomyosarcoma in 1854 , a clear histologic definition was not available until 1946, when Stout recognized the distinct morphology of Rhabdomyoblast. Stout described Rhabdomyoblasts as appearing in round, strap, racquet, and spider forms. As its name suggests, the tumor arise from a primitive muscle cells ${ }^{2}$.Although the tumor is believed to arise

a. Dr. Most. Umme Habiba Begum, MBBS, FCPS (Pediatrics), Honorary Medical officer, Pediatric Hemato-oncology dept., BSMMU, Dhaka

b. Prof. Afiqul Islam, MBBS, FCPS, MD, Professor and Chairman, Department of Pediatric Hematology and, Oncology BSMMU, Dhaka, Bangladesh

Address of Correspondence: Dr Most. Umme Habiba Begum, Honorary Medical officer, Pediatric Hemato-oncology dept., BSMMU, Dhaka, e-mail: arafanis@yahoo.com Mobile: 01197119945, 01191295626, 01712546647

Received: 11 September, 2011

Accepted: 11 June, 2012 dysphagia, chronic otorrhea, dysuria, haematuria, and proptoses were $10 \%$ each ( $n=02$, each); The histological sub-types were Embryonal $60 \%(n=12)$, alveolar 30\% $(n=6)$, and Botryoid 10\% (n=02); Of Embryonal variety in head and neck region $58.33 \%(n=7)$, and Genito-urinary sites 41.67\% (n=5); of Alveolar variety in trunk $66.67 \%(n=4)$, and in extremities33.33\% ( $n=2)$, of Botryoid sub-type frequency was equal in head - neck region and genitourinary site $50 \%$ each $(n=1)$.

Conclusion: Children with Rhabdomyosarcoma presented mostly in 1 to 5 years of age, with mass lesion (100\%), predominantly in head and neck region (40\%) and the commonest histological sub-type was Embryonal variety (60\%).

Key words: Rhabdomyosarcoma, clinicopathological profile, histological sub-types.

(J Bangladesh Coll Phys Surg 2012; 30: 132-136)

from primitive muscle cells, it can occur anywhere in the body except bone. The most common sites of Rhabdomyosarcoma in children are the head and neck region (35\%), the genitourinary tract (35\%) and the extremities (17\%). The orbit is the primary site in about $10 \%$ of these tumors; the most common localization in the head and neck area is parameningeal, including the nasopharynx, the paranasal sinuses, the middle ear and mastoid and the infratemporal fossa or pterygopalatine space. Most children are younger than 10 years of age $(72 \%){ }^{3}$

Rhabdomyosarcoma may be presented as a wide range of clinical features depending on the site of primary tumor. The most common presenting feature is a mass that may or may not be painful. Tumor arising from extremities may be presented with asymptomatic mass. But symptoms from other site involvement are caused by displacement or obstruction of normal structures. Tumors originating in the nasopharynx may be associated with nasal congestion, mouth breathing, 
epistaxis, and difficulty with swallowing and chewing. Rhabdomyosarcoma extended into the cranium can produce cranial nerve palsies, blindness and signs of increased intracranial pressure with headache and vomiting and that in the face or cheek may present with swelling, pain, and trismus. Tumor in the neck can produce progressive swelling with neurological symptoms after regional extension. Orbital primary tumor usually diagnosed with periorbital edema, proptosis, change in visual acuity, and local pain. When the tumor arises in middle ear, the most common early signs are pain, hearing loss, chronic otorrhea, or external ear mass. Rhabdomyosarcoma arising from urogenital tract may present with recurrent urinary tract infection or hematuria. ${ }^{4}$

Rhabdomyosarcoma is defined as demonstrating at least minimal evidence of Rhabdomyogenesis, or skeletal muscle differentiation. However, in a large proportion of cases, morphological evidence of myogenesis is limited to a small percentage of tumor cells or may be extremely difficult to detect. The use of antibodies for immunohistochemical detection of myogenesisassociated proteins such as desmin, myogenin (MYOG), and MyoD (MYOD1) have aided the diagnostic workup of such cases, and when combined myogenin and MyoD have $97 \%$ sensitivity to detect Rhabdomyosarcoma. The identification of muscle related differentiation is key and clinically relevant because some Rhabdomyosarcoma cases can be virtually indistinguishable from the group of so-called undifferentiated or non-Rhabdomyosarcoma soft-tissue sarcomas. ${ }^{5}$

Two major forms of the disease are described, conventionally termed Embryonal Rhabdomyosarcoma (ERMS) and Alveolar Rhabdomyosarcoma (ARMS), reflecting morphological similarities to fetal muscle or pulmonary alveoli, respectively. These distinctions are clinically relevant because the embryonal form typically shows less aggressive clinical behavior and a better prognosis. There is an embryonal variant known as spindle or botryoid tumors which are highly curable. When clinical stages and other variables are taken into account, survival rates range from $90 \%$ in localized form of embryonal Rhabdomyosarcoma to as low as $20 \%$ for patients with metastatic alveolar histologic tumors.

Though Rhabdomyosarcoma is a very common soft tissue sarcoma in children, there is no published literature of clinicopathological profile of this tumor in our country. The findings of this study could help the health professionals of our country in early diagnosis of Rhabdomyosarcoma and initiating treatment with a view of rewarding outcome.

\section{Materials and methods:}

The study was a hospital based prospective observational study, carried out in the department, of paediatric Hemato- Oncology, Bangabandhu Sheikh Mujib Medical University (BSMMU), Dhaka in between January to December 2009. Total 20 diagnosed cases of Rhabdomyosarcoma in children aged 1 to 15 years, those attending in and out patient department of the hospital were enrolled in this study. Children aged over 15 years and soft tissue sarcomas other than Rhabdomyosarcomas were excluded from the study.

There were no ethical problems, as before study procedure conducted, verbal consent of patients' attendants and also from the patients was taken. Disagreed cases were not included in this study.

Data was collected from in and outpatient departments of Bangabandhu Sheikh Mujib Medical University (BSMMU) Dhaka. Cases were selected according to inclusion and exclusion criteria. Face to face interviews were taken. Relevant informations (according to questionnaire) were taken from attendants preferably from parents and patients (10 years and older). In the history of illness, clinical presentation of the tumor particularly related to primary sites, and presenting physical problems were analyzed. Physical examinations were done in detail. During examination, emphasis was given on identifying primary tumor site. In all cases available documents were examined to see investigation reports particularly imaging and histopathological reports to correlate clinical presentations with histological sub-types.

After data collection all data was entered in master sheet and analyzed manually in view of the objective of the study. Frequency, distribution and proportions were calculated for the values. Results were published in graph and tabulated form.

\section{Results:}

Total 20 cases were included in this study and following results were found. 
Table- 1 shows Age distribution. Mean age was 6.83 years, and standard deviation was 4.41. Minimum age was 5 months and maximum age was 13 years. Maximum number of patients were in 1-5 years age group ( $n=9,45 \%)$.

\section{Table-I}

Age distribution $(n=20)$

\begin{tabular}{lcc} 
Age in years & Number of patients & Percentage (\%) \\
\hline $0-1$ & 03 & $15 \%$ \\
$1-5$ & 09 & $45 \%$ \\
$5-10$ & 04 & $25 \%$ \\
$10-15$ & 06 & $40 \%$ \\
\hline Total & $=20$ & $100 \%$ \\
\hline
\end{tabular}

Figure-1shows sex distribution. Among all the patients male was $85 \%(n=17)$ and female was $15 \%(n=03)$ with male to female ratio 5.66:1.
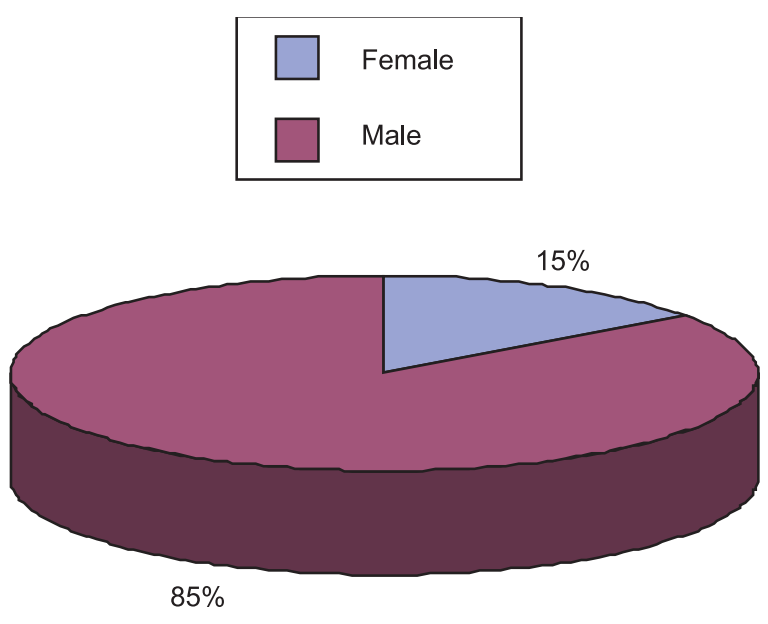

Fig.-I: Pie chart showing sex distribution

Table-ll summarizes Anatomical Location of primary tumor. Most common anatomical location of primary tumor was in head and neck region $40 \%(n=08)$. The next common sites were genito-urinary $30 \%(\mathrm{n}=06)$, extremity $20 \%(\mathrm{n}=04)$, and trunk $10 \%(\mathrm{n}=02)$. In the head and neck region, orbit $15 \%(\mathrm{n}=03)$, ear $10 \%$ $(\mathrm{n}=02)$, nasopharynx $05 \%(\mathrm{n}=01)$, paranasal sinuses 05 $\%(\mathrm{n}=01)$, tongue $05 \%(\mathrm{n}=01)$.
Table II

\begin{tabular}{|c|c|c|c|}
\hline \multicolumn{4}{|c|}{ Anatomical Location of primary tumor } \\
\hline Anatomical location & Frequency & & Percent (\%) \\
\hline Head and neck & 08 & & $40 \%$ \\
\hline Orbit & 03 & & $15 \%$ \\
\hline Nasopharyx & & 01 & $05 \%$ \\
\hline Ear & 02 & & $10 \%$ \\
\hline Paranasal sinuses & 01 & & $05 \%$ \\
\hline Tongue & 01 & & $05 \%$ \\
\hline Genito- urinary & 06 & & $30 \%$ \\
\hline Urinary bladder & 03 & & $15 \%$ \\
\hline Testes & 03 & & $15 \%$ \\
\hline Extremity & 04 & & $20 \%$ \\
\hline Lower limb & 04 & & $20 \%$ \\
\hline Trunk & 02 & & $10 \%$ \\
\hline Total & $=20$ & & $=100 \%$ \\
\hline
\end{tabular}

Table-lll summarizes Clinical presentations. The most common presenting feature was primary tumor mass $100 \%(n=20)$ irrespective of sites. Other presenting features were local pain $25 \%(n=05)$, urinary obstruction $15 \%(n=03)$, and dysphagia, otorrhea, proptosis, haematuria, dysuria $10 \%$ each ( $n=02)$; epistaxis, ocular palsy, and nasal obstruction 5\% ( $\mathrm{n}=01)$.

Figure ll- shows distribution of histopathological subtypes of Rhabdomyosarcoma. The Embryonal Rhabdomyosarcoma was the most common histological sub-type $60 \%(n=12$,). Other types were Alveolar subtype $30 \%(n=06)$, and Botryoid sub- type of embryonal variety10\% $(n=02)$; No pleomorphic or anaplastic variety was found.

Table-lV summarizes Clinical presentations in relation to pathological -subtype. Among 12 cases of Embryonal variety 7 was found in head and neck region (58.33\%), 5 was found in genito-urinary tumors (41.66\%), among total 6 cases of Alveolar variety 4 was found in trunk (66.66\%) and 2 in extremities (33.33\%), total 2 cases were of Botryoid sub-type, among them 1 was from head and neck region, other one from genitourinary site. 
Table-Ill

\section{Clinical presentations}

\begin{tabular}{|c|c|c|c|c|}
\hline \multicolumn{2}{|c|}{ Anatomical location } & \multirow{2}{*}{$\begin{array}{l}\text { Clinical presentation } \\
\text { Tumor mass }\end{array}$} & \multirow{2}{*}{$\frac{\text { Frequency }}{03}$} & \multirow{2}{*}{$\begin{array}{c}\text { Percentage } \\
15 \%\end{array}$} \\
\hline Head and neck & Orbit & & & \\
\hline & & Proptosis & 02 & $10 \%$ \\
\hline & & Ocular palsy & 01 & $05 \%$ \\
\hline & & Local Pain & 01 & $05 \%$ \\
\hline & Nasopharyx & Tumor mass & 01 & $05 \%$ \\
\hline & & Dysphagia & 01 & $05 \%$ \\
\hline & & Epistaxis & 01 & $05 \%$ \\
\hline & Ear & External ear mass & 02 & $10 \%$ \\
\hline & & Local pain & 02 & $10 \%$ \\
\hline & & Otorrhoea & 02 & $10 \%$ \\
\hline & Paranasal sinuses & Tumor massNasal obstruction & 0101 & $05 \% 05 \%$ \\
\hline & Tongue & Tumor mass & 01 & $05 \%$ \\
\hline & & Dysphagia & 01 & $05 \%$ \\
\hline \multirow[t]{6}{*}{ Genito-urinary } & Urinary bladder & Tumor mass & 03 & $15 \%$ \\
\hline & & Haematuria & 02 & $10 \%$ \\
\hline & & Dysuria & 02 & $10 \%$ \\
\hline & & Urinary obstruction & 03 & $15 \%$ \\
\hline & Testes & Tumor mass & 03 & $15 \%$ \\
\hline & & Local pain & 02 & $10 \%$ \\
\hline Extremities & Lower limb & Tumor mass & 04 & $20 \%$ \\
\hline Trunk & & Tumor mass & 02 & $10 \%$ \\
\hline
\end{tabular}

Table-IV

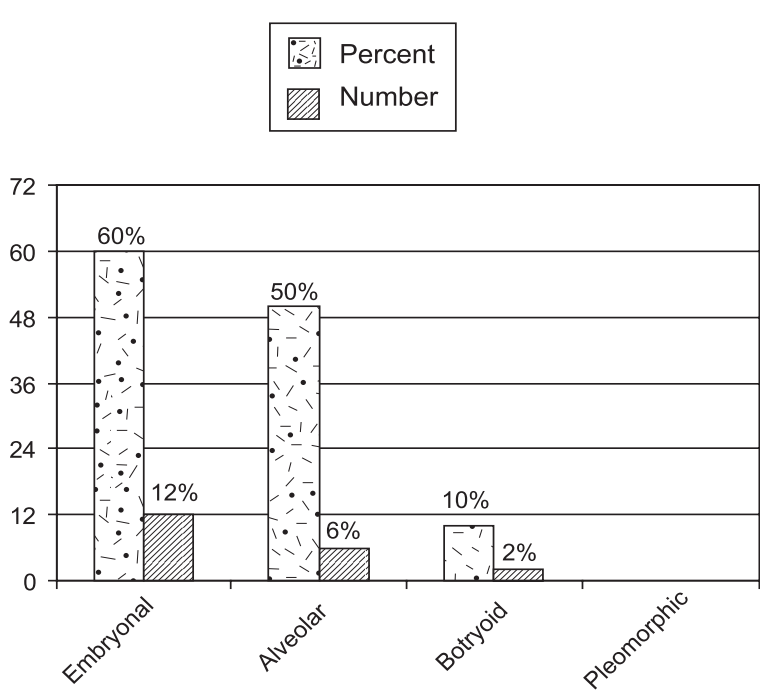

Fig.-2: Bar diagram showing histopathological subtypes of Rhabdomyosarcoma $(n=20)$

\begin{tabular}{lcccc}
\multicolumn{5}{c}{$\begin{array}{c}\text { Clinical presentations in relation to } \\
\text { pathological -subtypes }\end{array}$} \\
Primary sites & $\begin{array}{l}\text { Embryonal } \\
\text { (Number) }\end{array}$ & $\begin{array}{c}\text { Alveolar } \\
\text { (Number) }\end{array}$ & (Number) & (Number) \\
\hline Head and neck RMS & 7 & 0 & 1 & 0 \\
Genito-urinary RMS & 5 & 0 & 1 & 0 \\
Trunk RMS & 0 & 4 & 0 & 0 \\
Extremity RMS & 0 & 2 & 0 & 0 \\
\hline Total & 12 & 6 & 2 & 0 \\
\hline
\end{tabular}

\section{Discussion:}

This hospital based prospective observational study showed that the peak incidence of Rhabdomyosarcoma was in 1-5 years of age group ( $n=9,45 \%)$ with mean age 6.83 years, though the age range was 5 months to 13 years and male to female ratio was 5.66: 1(17:3). In a study by Brown BJ et al. at the University College Hospital Ibadan, Nigeria (between 1984 and 2003) 
showed mean age of presentation of Rhabdomyosarcoma was found 6.2(4.1) years. ${ }^{6}$ The highest incidence of tumors was found in head and neck region $(40 \%, n=08)$, among them orbit $15 \%(n=03)$, nasopharynx 05\% $(n=01)$, ear $10 \%(n=02)$, paranasal sinuses $05 \%(n=01)$, and tongue $05 \%(n=01)$. The next common incidence of Rhabdomyosarcoma was found in genito-urinary tract, 30\% $(n=06)$ among them urinary bladder $15 \%(n=03)$, testes $15 \%(n=03)$. Tumor incidence in extremities was $20 \%(n=04)$. The least incidence of the tumor was in trunk $10 \%(n=02)$. Brown BJ et al. observed the majority (50.6\%) of tumors was in the head and neck region and the common primary sites were soft tissue of the head, face (24.2\%) and orbit (14.3\%). Other sites included soft tissue of the pelvis $(11.0 \%)$, genito-urinary tract $(9.9 \%)$ and abdomen (9.9\%). ${ }^{6}$ The variation of incidence the tumor with other international studies regarding primary tumor site may be due to small sample size.

In the current study tumor mass was found in $100 \%(n=20)$ cases, most lesions of limbs and trunks were painless; only in $25 \%(n=05)$ cases located in ear and orbit lesions were associated with local pain. Urinary retention was the next common presenting feature $(n=03,15 \%)$ in our study. Urinary retention was due to involvement of urinary bladder leading to bladder outflow obstruction. Proptosis was observed in $10 \%$ ( $n=02)$ cases. It was due to tumor growth in retro orbital space and associated with ocular palsy ( $n=01,05 \%)$.Chronic otorrhea was found in $10 \%(n=02)$ cases whose were due to middle ear involvement of Rhabdomyosarcoma leading to chronic otitis media. Dysuria and haematuria was found in $10 \%(n=02)$ cases whose were associated with urinary bladder involvement and urinary obstruction. Dysphagia was observed in some tumors $10 \%(\mathrm{n}=02)$ involving tongue and nasopharynx. The least manifestation was nasal obstruction, observed in $05 \%(n=1)$ cases. Epistaxis was another uncommon presentation. Rhabdomyosarcoma involving nasopharynx was presented with the same. There was no feature of raised intracranial pressure in any case.

In our study, we got almost all histological sub-types of Rhabdomyosarcoma except pleomorphic one. The embryonal sub-type was found $60 \%(n=12)$, and alveolar sub-type $30 \%(n=06)$ cases. Botryoid sub-type was found in $10 \%(n=02)$ cases of whose mostly found in genitourinary tract tumor. This finding is almost consistent with the finding observed by Pappo AS et al. who observed the incidence of Embryonal Rhabdomyosarcoma in 55\% of patients; the Botryoid variant in $5 \%$ of patients and Alveolar Rhabdomyosarcoma in $20 \%$ of patients. ${ }^{7}$ In current study, of Embryonal variety more was found in head and neck region ( $\mathrm{n}=7,58.33 \%)$, and also in Genitourinary sites ( $n=5,41.67 \%$ ); on the other hand, of Alveolar variety more was found in trunk $(n=4,66.67 \%)$ and also in extremities ( $n=2,33.33 \%)$; of Botryoid sub-type frequency was equal in head and neck region and genitourinary sites ( $\mathrm{n}=1$ each, 50\%). Parham et al. has shown that Embryonal subtype is the most frequently observed Rhabdomyosarcoma in children (60\% to $70 \%$ ) and typically arise in the head and neck region or in the genitourinary tract; Botryoid tumors represent about $10 \%$ of all Rhabdomyosarcoma cases that arise under the mucosal surface of body orifices such as the vagina, bladder, nasopharynx, and biliary tract; approximately 20\% is alveolar subtype with primary sites involving the extremities, trunk, and perineum or perianal region; Pleomorphic one occurs rarely in children. ${ }^{8}$

\section{Conclusion:}

In this study, children with Rhabdomyosarcoma presented mostly in 1 to 5 years of age with male to female ratio 5.66: 1(17:3); predominant lesions were in head and neck region (40\%) followed by genitourinary sites (30\%) and extremity (20\%). Common presentations were mass lesion (100\%), local Pain (25\%), urinary obstructions (15\%), proptosis, dysphagia, otorrhea, and haematuria (10\% each). The most common histological sub-type was Embryonal (60\%), followed by Alveolar (30\%) and Botryoid (10\%).

\section{References:}

1. Arndt CA, Crist WM. Common musculoskeletal tumors of childhood and adolescence. N Engl J Med. Jul 29 1999; 341(5):342-52.

2. Stout AP. Rhabdomyosarcoma of the skeletal muscles. Ann Surg. 1946; 123: 447-72.

3. Stephanie E Combs, Wolfgang Behsnich, Andeas E Kulozick, Peter E Huber, Jurgen Debus and Daniela Schulz -Ertner: Intensity Modulated Radiotherapy and Fractionated Stereotactic Radiotherapy for children with head-and-neckrhabdomyosarcoma; BMC Cancer. 2007; 7: 177

4. Carola A, S. Arndt: Soft tissue sarcoma; Nelson Textbook of Pediatrics $18^{\text {th }}$ edition 2007; part 21; chapter 500; 2144-45.

5. Elai Davicioni, Michael J. Anderson, Friedrich Graf Finckenstein, James C. Lynch, Stephen J, Qualman Hiroyuki Shimada, et al: Molecular Classification of Rhabdomyosarcoma-Genotypic and Phenotypic Determinants of Diagnosis ; American Journal of Pathology. 2009;174: 550-564.

6. Brown BJ; Oluwasola AO: Childhood rhabdomyosarcoma in Ibadan, Nigeria: 1984-2003.Ann Trop Paediatr. 2006; 26(4):349-55

7. Pappo AS, Shapiro DN, Crist WM, Maurer HM. Biology and therapy of pediatric rhabdomyosarcoma. J Clin Oncol. Aug 1995;13 (8):2123-39.

8. Parham DM, Ellison DA: Rhabdomyosarcomas in adults and children: an update. Arch Pathol Lab Med 130 (10): 145465, 2006. 